\title{
Seletividade de formulações de nicosulfuron para híbridos de milho em função da época da adubação nitrogenada ${ }^{1}$
}

\author{
Selectivity of nicosulfuron formulations for corn hybrids in function of nitrogen \\ fertilization period
}

\author{
Naiara Guerra ${ }^{2}$, Antonio Mendes de Oliveira Neto ${ }^{2}$, Cleber Daniel de Goes Maciel ${ }^{3}$, Juliana \\ Parisotto Poletine ${ }^{3}$, Gesley Ramos Guimarães Lima ${ }^{4}$, Luis Carlos Sola Júnior ${ }^{4}$
}

\begin{abstract}
Resumo - Objetivou-se no presente trabalho avaliar o efeito da interação entre as épocas de adubação nitrogenada de cobertura e diferentes formulações do herbicida nicosulfuron sobre a seletividade aos híbridos de milho 2B-710 e 30K73. Dois experimentos foram conduzidos simultaneamente, com plantas de milho em estádios $\mathrm{V}_{3}$ e $\mathrm{V}_{6}$, sendo os tratamentos dispostos em esquema fatorial $2 \times 4+1$, onde o primeiro fator foi constituído por duas formulações de nicosulfuron $\left(18,8 \mathrm{~g} \mathrm{ha}^{-1}\right)\left(\right.$ Sanson $\left.^{\circledR} \mathrm{e} \mathrm{Accent}^{\circledR}\right)$ em mistura com atrazine $\left(1250 \mathrm{~g} \mathrm{ha}^{-1}\right)$; o segundo fator por quatro épocas de adubação nitrogenada $\left(67,5 \mathrm{~kg} \mathrm{~N}^{-1}\right)$, representadas por $0,3,6$ e 9 dias após a aplicação do herbicida (DAAH); mais uma testemunha adicional sem aplicação de herbicidas. Os híbridos de milho 2B-710 e 30K73 apresentaram seletividade variável quanto à aplicação de nicosulfuron, sendo que os maiores efeitos e sintomas da fitointoxicação ocorreram quando a adubação nitrogenada foi realizada em cobertura no mesmo dia da aplicação da formulação Sanson ${ }^{\circledR}$ para o estádio $\mathrm{V}_{3}$, e aos três dias da aplicação de $A$ ccent ${ }^{\circledR}$ para o estádio $\mathrm{V}_{6}$.
\end{abstract}

Palavras-chave: Herbicida, fitointoxicação, nitrogênio

\begin{abstract}
Aimed in the present work to evaluate the interaction effect between periods of nitrogen coverage fertilization and different formulations of nicosulfuron herbicide over the selectivity to 2B-710 and 30K73 corn hybrids. Two experiments were conducted, simultaneously, with corn seedlings in $V_{3}$ and $V_{6}$ stages using treatments disposed in a factorial scheme $2 \times 4+1$, where the first factor was composed by two nicosulfuron formulations $\left(18.8 \mathrm{~g} \mathrm{ha}^{-1}\right)\left(\right.$ Sanson $^{\mathrm{TM}}$ and Accent $\left.{ }^{\mathrm{TM}}\right)$ in mixture with atrazine $\left(1250 \mathrm{~g} \mathrm{ha}^{-1}\right)$ and the second factor constituted by four periods of nitrogen coverage fertilization $\left(67.5 \mathrm{~kg} \mathrm{~N} \mathrm{ha}^{-1}\right)$, represented by $0,3,6$ and 9 days after herbicide application (DAAH); and an additional check without herbicide application. 2B-710 and $30 \mathrm{~K} 73$ corn hybrids showed variable selectivity in relation to nicosulfuron herbicide application, with the major effects and symptoms of phytotoxicity occurring when nitrogen fertilization was performed in coverage in the same day of Sanson ${ }^{\mathrm{TM}}$ formulation applying for $\mathrm{V}_{3}$ stage and three days from applying Accent $^{\mathrm{TM}}$ to $\mathrm{V}_{6}$ stage.
\end{abstract}

\footnotetext{
* Autor para correspondência

${ }^{1}$ Recebido para publicação em 08/01 2011 e aceito20/02/2011.

${ }^{2}$ Discente do Curso de Pós-Graduação em Agronomia na área de Proteção de Plantas, NAPD/UEM, E-mail: naiara.guerra@hotmail.com;

${ }^{3}$ Eng $^{\circ}$-Agro., D.Sc. Professor Adjunto, Depto de Ciências Agronômicas - Universidade Estadual de Maringá, UEM. Campus Avançado de Umuarama/PR - CAU/CCA. Estrada da Paca s/n, 87507-190, Umuarama/PR.

${ }^{4}$ Discentes do curso de Agronomia da Escola Superior de Agronomia de Paraguaçu Paulista/SP, ESAPP.
} 
Guerra et al.

Key-words: Herbicide, phytotoxicity, nitrogen

\section{Introdução}

A aplicação de herbicidas em pósemergência na cultura do milho surgiu e se fortaleceu como uma ferramenta para o controle de plantas daninhas (Christoffoleti \& Mendonça, 2001). Contudo, segundo LópezOvejero et al. (2003a), a adoção desta técnica de manejo, requer observação de alguns fatores, tais como o híbrido utilizado, o estádio fenológico da cultura no instante da aplicação e o intervalo entre aplicação do herbicida e do inseticida organofosforado e/ou da adubação nitrogenada de cobertura, que, quando negligenciados, podem interferir em sua seletividade e causar intoxicação à cultura (López-Ovejero et al., 2003b).

O nicosulfuron 2 dimetoxipirimidina. 2.il-carbamoilsulfamoil) $\mathrm{N}, \mathrm{N}$. imetilnicoti-namida, é um herbicida sistêmico do grupo químico das sulfoniluréias, que se destaca entre os principais pósemergentes utilizados na cultura do milho, principalmente para o controle de gramíneas e algumas dicotiledôneas (Rodrigues \& Almeida, 2005). Os herbicidas deste grupo inibem a acetolactato sintase (ALS), primeira enzima comum à rota de biossíntese de aminoácidos de cadeia ramificada, valina, leucina e isoleucina, provocando a interrupção da síntese de proteínas, e consequente inibição da divisão celular e crescimento da planta (Silva \& Silva, 2007; Victória Filho, 2008).

A aplicação de nitrogênio em cobertura tem importante função no desenvolvimento da cultura, uma vez que é o nutriente absorvido em maior quantidade pelo milho, além de ser constituinte de proteínas, ácidos nucléicos, citocromos e clorofila (Cantarella et al., 2005; Costa et al., 2005). Sendo então um dos principais fatores responsáveis pela expressão do potencial máximo de rendimento da cultura (Rizzardi et al., 2008).
Em geral, a adubação de cobertura tem sido realizada quando as plantas de milho apresentam de 3 a 5 folhas totalmente expandidas (Fancelli \& Dourado-Neto, 2000), época próxima a recomendada para aplicação do herbicida nicosulfuron (2-6 folhas totalmente expandidas) (Spader \& Vidal, 2001; Silva et al., 2004).

Segundo Peixoto \& Ramos (2002), para aplicação do nicosulfuron são necessários intervalos de aplicação de 7 a 10 dias antes e após a aplicação de adubos nitrogenados. Para Devine et al. (1993), determinados herbicidas influenciam algumas rotas metabólicas, sendo que essas estão direta ou indiretamente relacionadas ao metabolismo do nitrogênio. Nicolai et al. (2006), estudando o efeito da interação entre os herbicidas pós-emergentes e épocas de adubação nitrogenada, verificaram fitointoxicação inicial nas plantas de milho quando a adubação de cobertura foi realizada no mesmo dia ou próximo da aplicação dos herbicidas.

O presente trabalho teve como objetivo avaliar a interferência inicial de épocas de adubação nitrogenada de cobertura, sobre a seletividade de formulações do herbicida nicosulfuron aos híbridos de milho 2B-710 e $30 \mathrm{~K} 73$, em dois estádios de desenvolvimento da cultura $\left(\mathrm{V}_{3}\right.$ e $\left.\mathrm{V}_{6}\right)$.

\section{Material e métodos}

Os quatro experimentos foram conduzidos em estufas plásticas do campus urbano da Escola Superior de Agronomia de Paraguaçu Paulista/SP - ESAPP, localizado no município de Paraguaçu Paulista-SP, cujas coordenadas geográficas são latitude em $22^{\circ} 34^{\prime} 53^{\prime \prime} \mathrm{S}$, longitude de $50^{\circ} 34^{\prime} 35^{\prime \prime} \mathrm{W}$ e altitude média de 506 metros. O período de condução dos experimentos foi de 29 de janeiro a 26 de fevereiro de 2008. 
Guerra et al.

O solo utilizado para o preenchimento das unidades experimentais foi coletado a uma profundidade 0 a $20 \mathrm{~cm}$ de um Latossolo Vermelho distroférrico, de textura arenosa, constituído por $80,3 \%$ de areia; $12,2 \%$ de argila e 7,5\% de silte. A análise química deste solo apresentou $\mathrm{pH}$ de 5,5 em $\mathrm{CaCl}_{2}, 17 \mathrm{mmol}_{\mathrm{c}} \mathrm{dm}^{-3}$ de $\mathrm{H}^{+}+\mathrm{Al}^{3+} ; 32,0 \mathrm{mmol}_{\mathrm{c}} \mathrm{dm}^{-3}$ de $\mathrm{Ca}^{+2} ; 9,0$ $\mathrm{mmol}_{\mathrm{c}} \mathrm{dm}^{-3}$ de $\mathrm{Mg}^{+2} ; 1,3 \mathrm{mmol}_{\mathrm{c}} \mathrm{dm}^{-3}$ de $\mathrm{K}^{+}$; $32,0 \mathrm{mg} \mathrm{dm}^{-3}$ de P; 12,0 $\mathrm{g} \mathrm{dm}^{-3}$ de MO; SB de 42,0 $\mathrm{mmol}_{\mathrm{c}} \mathrm{dm}^{-3}$; CTC de 59,0 $\mathrm{mmol}_{\mathrm{c}} \mathrm{dm}^{-3} \mathrm{e}$ V\% de $71,0 \%$.

$\mathrm{O}$ delineamento experimental utilizado foi $o$ inteiramente casualizado, com cinco repetições. As unidades experimentais foram representadas por vasos de polietileno com capacidade de $8 \mathrm{dm}^{3}$ de solo, onde foram semeadas em duas etapas, intercaladas por dez dias quatro sementes de milho por vaso. Após a emergência do milho, foi realizado o desbaste permanecendo apenas duas plantas por unidade experimental, representando os estádios fenológicos $V_{3}$ e $V_{6}$, no momento da aplicação dos herbicidas.

Os tratamentos foram dispostos em esquema fatorial $2 \times 4+1$, onde o primeiro fator foi constituído por duas formulações do herbicida nicosulfuron $\left(18,8 \mathrm{~g} \mathrm{ha}^{-1}\right)\left(\right.$ Sanson $^{\circledR} \mathrm{e}$ Accent $\left.{ }^{\circledR}\right)$ em mistura com atrazine $\left(1250 \mathrm{~g} \mathrm{ha}^{-}\right.$ ${ }^{1}$ ), e o segundo por quatro épocas de adubação nitrogenada, representada por $0,3,6$ e 9 dias após a aplicação do herbicida (DAAH) e uma testemunha adicional sem aplicação de herbicida, mas com aplicação de nitrogênio parcelada aos 3 e 6 DAAH. A fonte nitrogenada utilizada foi a uréia, sendo esta incorporada ao solo a $5 \mathrm{~cm}$ de profundidade, em dose correspondente a $150 \mathrm{~kg} \mathrm{ha}^{-1}(67,5 \mathrm{~kg}$ $\mathrm{N} \mathrm{ha}^{-1}$ ).

A aplicação dos tratamentos herbicidas foi realizada com um pulverizador costal pressurizado a $\mathrm{CO}_{2}$, utilizando pressão constante de $207 \mathrm{kPa}$ e barra de quatro pontas de jato plano DG110.02 VS, posicionada a 50 cm de altura do alvo biológico, com volume de calda de $200 \mathrm{~L} \mathrm{ha}^{-1}$. No momento da aplicação os dados climatológicos médios foram: temperatura do ar de $28^{\circ} \mathrm{C}$, umidade relativa de $83 \%$, velocidade do vento foi de $2 \mathrm{~km} \mathrm{~h}^{-1}$, sendo que o céu encontrava-se com poucas nuvens e o solo das unidades experimentais em capacidade de campo.

A fitointoxicação nos híbridos de milho foi avaliada aos 3, 7 e 14 dias após aplicação (DAA), por meio de escala percentual de danos visuais (SBCPD, 1995). Além disso, também foram avaliados os parâmetros: teores de clorofila na folha, medidos com o clorofilômetro SPAD, modelo Minolta ${ }^{\circledR}$; altura das plantas, com auxílio de régua milimetrada, aos 3, 7 e 14 DAA, e massa seca da parte aérea e das raízes aos 14 DAA. Para determinação da massa seca, a parte aérea e raízes dos híbridos foram inicialmente coletadas, separadamente e embaladas em sacos de papel, e sendo levados posteriormente a estufa de circulação de ar forçada a $70^{\circ} \mathrm{C}$ por 12 horas. Após a retirada do material da estufa, foram tomados a massa seca por pesagem em balança de precisão.

Os dados de teor de clorofila, altura e massa seca da parte aérea e raiz foram submetidos à análise de variância pelo teste $\mathrm{F}$, e comparados pelo teste de Tukey, a 5\% de probabilidade. A comparação das médias dos tratamentos em relação à testemunha foi realizada pelo teste de Dunnett a $5 \%$ de probabilidade.

\section{Resultados e discussão}

Híbridos em Estádio $V_{3}$

Os sintomas de fitointoxicação nas plantas de milho em estádio $\mathrm{V}_{3}$ se caracterizaram por clorose e enrugamento das lâminas das folhas novas em expansão, observados aos 3 dias após aplicação das formulações de nicosulfuron. Posteriormente, com o desenvolvimento das folhas, os sintomas 
Guerra et al.

passaram para parte central da lâmina em forma de manchas cloróticas.

Os maiores níveis de fitointoxicação ocorreram quando a adubação nitrogenada foi realizada no mesmo dia da aplicação do herbicida nicosulfuron (0 DAAH) (Tabela 1). A adubação nitrogenada realizada 0 DAAH com a formulação Sanson ${ }^{\circledR}$ promoveu maiores danos visuais e de forma mais precoce quando comparado a formulação Accent ${ }^{\circledR}$, nos dois híbridos.

Além disso, apesar do Sanson $^{\circledR}$ e Accent $^{(\mathbb{B}}$ apresentarem o mesmo ingrediente ativo em suas formulações, os resultados demonstraram comportamento distinto de sensibilidade para os híbridos, caracterizados aos 14 DAA pelo Sanson ${ }^{\circledR}$, quando os sintomas de fitointoxicação visual foram superiores aos provocados pelo Accent ${ }^{\circledR}$. Nicolai et al. (2006) também verificaram sintomas de fitointoxicação quando o híbrido AGN 2012 recebeu a adubação nitrogenada no mesmo dia da aplicação de nicosulfuron + atrazine no estádio $\mathrm{V}_{4}-\mathrm{V}_{5}$, entretanto, nas avaliações subseqüentes perceberam que as plantas tiveram capacidade para detoxificar as moléculas de herbicida absorvidas, havendo redução desses sintomas. Mascarenhas (2004) testou a aplicação no mesmo dia de uréia e mesotrione + atrazine + óleo e verificou fitointoxicação inicial, porém, sem redução no rendimento dos grãos, quando comparado com a testemunha capinada.

Tabela 1. Fitointoxicação visual (\%) dos híbridos de milho 2B-710 e 30K73 e aplicação no estádio de fenológico $V_{3}$, em função das formulações de nicosulfuron Sanson ${ }^{\circledR}$ e Accent ${ }^{\circledR}$ e diferentes épocas de adubação nitrogenada.

\begin{tabular}{|c|c|c|c|c|c|c|}
\hline \multirow{3}{*}{$\begin{array}{c}\text { Época de } \\
\text { adubação } \\
\text { (DAA) }\end{array}$} & \multicolumn{6}{|c|}{ Fitointoxicação (\%) - híbrido 2B-710 } \\
\hline & \multicolumn{2}{|c|}{$3 \mathrm{DAA}$} & \multicolumn{2}{|c|}{$7 \mathrm{DAA}$} & \multicolumn{2}{|c|}{14 DAA } \\
\hline & Sanson $^{\circledR}$ & Accent ${ }^{\circledR}$ & Sanson $^{(8)}$ & Accent ${ }^{(\circledast)}$ & Sanson $^{(8}$ & Accent $^{\circledR}$ \\
\hline 0 & 14,6 & 4,6 & $\overline{62,0}$ & $\overline{15,0}$ & 61,2 & $\overline{16,0}$ \\
\hline 3 & 4,2 & 4,2 & 0,0 & 0,0 & 0,0 & 0,0 \\
\hline 6 & 3,8 & 3,8 & 0,0 & 0,0 & 0,0 & 0,0 \\
\hline 9 & 3,8 & 2,4 & 0,0 & 0,0 & 0,0 & 0,0 \\
\hline \multirow[t]{2}{*}{ Testemunha } & 0,0 & 0,0 & 0,0 & 0,0 & 0,0 & 0,0 \\
\hline & \multicolumn{6}{|c|}{ Fitointoxicação (\%) - híbrido 30K73 } \\
\hline 0 & 7,0 & 7,8 & 29,4 & 7,8 & 31,0 & 25,0 \\
\hline 3 & 0,0 & 0,0 & 0,0 & 0,0 & 0,0 & 0,0 \\
\hline 6 & 0,0 & 0,0 & 0,0 & 0,0 & 0,0 & 0,0 \\
\hline 9 & 0,0 & 0,0 & 0,0 & 0,0 & 0,0 & 0,0 \\
\hline Testemunha & 0,0 & 0,0 & 0,0 & 0,0 & 0,0 & 0,0 \\
\hline
\end{tabular}

Para o teor de clorofila nas folhas, mensurado através do índice SPAD (Tabela 2), foi constatada maior sensibilidade dos híbridos a formulação $\operatorname{Sanson}^{\circledR}$ em relação ao Accent $^{\circledR}$, principalmente para 2B-710, quando a adubação nitrogenada foi realizada no mesmo dia da aplicação dos herbicidas (0 DAAH). Verificou-se também que o híbrido 2B-710 também apresentou maior sensibilidade inicial às formulações de nicosulfuron em relação à testemunha, uma vez que diferente do $30 \mathrm{~K} 73$, com exceção para o Accent ${ }^{\circledR}$, todas as épocas de adubação diferiram significativamente aos 3 DAA. 
Guerra et al.

Tabela 2. Teor de clorofila nas folhas (SPAD) dos híbridos de milho 2B-710 e 30K73 em aplicação no estádio de fenológico $\mathrm{V}_{3}$, em função das formulações de nicosulfuron Sanson ${ }^{\circledR} \mathrm{e}$ Accent $^{\circledR}$ e diferentes épocas de adubação nitrogenada.

\begin{tabular}{|c|c|c|c|c|c|c|}
\hline \multirow{3}{*}{$\begin{array}{l}\text { Época de } \\
\text { adubação } \\
\text { (DAA) }\end{array}$} & \multicolumn{6}{|c|}{ Teor de clorofila (SPAD) - híbrido 2B-710 } \\
\hline & \multicolumn{2}{|c|}{$3 \mathrm{DAA}$} & \multicolumn{2}{|c|}{$7 \mathrm{DAA}$} & \multicolumn{2}{|c|}{$14 \mathrm{DAA}$} \\
\hline & Sanson $^{\circledR}$ & Accent $^{\circledR}$ & Sanson $^{\circledR}$ & Accent $^{\circledR}$ & Sanson $^{\circledR}$ & Accent $^{(B)}$ \\
\hline$\overline{0}$ & $(*) 32,7 \mathrm{aB}$ & $\left({ }^{\mathrm{ns}}\right) 35,6 \mathrm{aA}$ & (*) 33,1 aA & $\left({ }^{\mathrm{ns}}\right) 36,5 \mathrm{aA}$ & $\left({ }^{\mathrm{ns}}\right) 33,2 \quad \mathrm{bB}$ & $\left({ }^{\mathrm{ns}}\right) 35,8 \mathrm{aA}$ \\
\hline 3 & (*) 33,1 aA & (*) $32,5 \mathrm{aA}$ & $\left({ }^{\mathrm{ns}}\right) 37,3 \mathrm{aA}$ & $\left({ }^{\mathrm{ns}}\right) 38,7 \mathrm{aA}$ & $\left({ }^{\mathrm{ns}}\right) 36,2 \mathrm{abA}$ & $\left({ }^{n s}\right) 35,6 \mathrm{aA}$ \\
\hline 6 & (*) $31,2 \mathrm{aA}$ & (*) $32,4 \mathrm{aA}$ & $\left({ }^{\mathrm{ns}}\right) 37,6 \mathrm{aA}$ & $\left({ }^{\mathrm{ns}}\right) 38,4 \mathrm{aA}$ & (ns) $35,8 \mathrm{abA}$ & $\left({ }^{n s}\right) 35,7 \mathrm{aA}$ \\
\hline 9 & (*) $31,8 \mathrm{aA}$ & (*) $31,8 \mathrm{aA}$ & $\left({ }^{\mathrm{ns}}\right) 35,5 \mathrm{aA}$ & $\left({ }^{\mathrm{ns}}\right) 37,9 \mathrm{aA}$ & $\left({ }^{n s}\right) 36,6$ a $A$ & $\left({ }^{n s}\right) 37,2$ aA \\
\hline Testemunha & \multicolumn{2}{|c|}{37,44} & \multicolumn{2}{|c|}{40,41} & \multicolumn{2}{|c|}{35,53} \\
\hline $\mathrm{CV}(\%)$ & \multicolumn{2}{|c|}{5,74} & \multicolumn{2}{|c|}{8,06} & \multicolumn{2}{|c|}{4,93} \\
\hline DMS $_{\text {herbicida }}$ & \multicolumn{2}{|c|}{2,46} & \multicolumn{2}{|c|}{3,84} & \multicolumn{2}{|c|}{2,26} \\
\hline $\mathrm{DMS}_{\text {adubação }}$ & \multicolumn{2}{|c|}{3,27} & \multicolumn{2}{|c|}{5,11} & \multicolumn{2}{|c|}{3,00} \\
\hline & \multicolumn{6}{|c|}{ Teor de clorofila (SPAD) - híbrido $30 \mathrm{~K} 73$} \\
\hline 0 & (*) $31,76 \mathrm{aB}$ & $\left({ }^{\mathrm{ns}}\right) 35,43 \mathrm{aA}$ & $\left({ }^{\mathrm{ns}}\right) 31,71 \mathrm{aA}$ & $\left({ }^{\mathrm{ns}}\right) 35,81 \mathrm{aA}$ & $\left({ }^{\mathrm{ns}}\right) 31,11 \mathrm{aA}$ & $\left({ }^{\mathrm{ns}}\right) 30,87 \mathrm{aA}$ \\
\hline 3 & $\left({ }^{\mathrm{ns}}\right) 33,87 \mathrm{aA}$ & (*) $31,77 \mathrm{aA}$ & $\left({ }^{\mathrm{ns}}\right) 37,85 \mathrm{aA}$ & $\left({ }^{\mathrm{ns}}\right) 36,42 \mathrm{aA}$ & $\left({ }^{\mathrm{ns}}\right) 31,61 \mathrm{aA}$ & $\left({ }^{\mathrm{ns}}\right) 39,00 \mathrm{aA}$ \\
\hline 6 & $\left({ }^{\mathrm{ns}}\right) 33,69 \mathrm{aA}$ & $\left({ }^{\mathrm{ns}}\right) 33,47 \mathrm{aA}$ & $\left({ }^{\mathrm{ns}}\right) 38,78 \mathrm{aA}$ & $\left({ }^{\mathrm{ns}}\right) 37,41 \mathrm{aA}$ & $\left({ }^{\mathrm{ns}}\right) 39,33 \mathrm{aA}$ & $\left({ }^{\mathrm{ns}}\right) 38,96 \mathrm{aA}$ \\
\hline 9 & $\left({ }^{\mathrm{ns}}\right) 33,24 \mathrm{aA}$ & $\left(n^{n s}\right) 33,14 a A$ & $\left(n^{\mathrm{ns}}\right) 37,54 \mathrm{aA}$ & $\left({ }^{\mathrm{ns}}\right) 35,54 \mathrm{aA}$ & $\left({ }^{\mathrm{ns}}\right) 27,83 \mathrm{aA}$ & $\left({ }^{\mathrm{ns}}\right) 33,18 \mathrm{aA}$ \\
\hline Testemunha & \multicolumn{2}{|c|}{35,99} & \multicolumn{2}{|c|}{40,05} & \multicolumn{2}{|c|}{39,02} \\
\hline $\mathrm{CV}(\%)$ & \multicolumn{2}{|c|}{6,88} & \multicolumn{2}{|c|}{17,73} & \multicolumn{2}{|c|}{30,16} \\
\hline DMS $S_{\text {herbicida }}$ & \multicolumn{2}{|c|}{2,97} & \multicolumn{2}{|c|}{8,37} & \multicolumn{2}{|c|}{13,34} \\
\hline $\mathrm{DMS}_{\text {adubação }}$ & \multicolumn{2}{|c|}{3,94} & \multicolumn{2}{|c|}{11,10} & \multicolumn{2}{|c|}{17,74} \\
\hline
\end{tabular}

Médias seguidas da mesma letra minúscula na coluna e maiúscula na linha não diferem entre si pelo teste de Tukey a 5\% de probabilidade. Médias representadas por “*” ou "ns” diferem ou não diferem, respectivamente, da testemunha sem herbicida, segundo o Teste de Dunnett a 5\% de probabilidade.

A altura de plantas do híbrido 2B-710 apresentou redução significativa aos 7 DAA para formulação Sanson ${ }^{\circledR}$ e aos 14 DAA para ambas formulações, quando a adubação nitrogenada foi realizada aos 0 DAAH (Tabela 3). Esse resultado também apresentou interação significativa através da análise do teste de Dunnett a $5 \%$ de probabilidade, quando os mesmos parâmetros de formulação e época de adubação foram comparados com a testemunha sem aplicação. Para o híbrido 30K73 não foram observadas diferenças significativas quanto à altura das plantas (Tabela 3).
Os valores de massa seca da parte aérea do híbrido 2B-710 confirmaram os resultados de fitointoxicação visual (\%), onde se observou que a adubação nitrogenada realizada no mesmo dia da aplicação de nicosulfuron $(0$ DAAH), nas formulações Sanson ${ }^{\circledR}$ e Accent $^{\circledR}$, apresentou menor acúmulo de massa seca aos 14 DAA (Tabela 4). A adubação nitrogenada realizada aos 0 DAAH Sanson ${ }^{\circledR}$ e Accent $^{\circledR}$ proporcionou redução do acúmulo de massa seca do híbrido 2B-710 em $84 \%$ e $49 \%$, respectivamente, confirmando a menor sensibilidade do híbrido 2B-710 a Sanson ${ }^{\circledR}$. 
Guerra et al.

Tabela 3. Altura média (cm) dos híbridos de milho 2B-710 e 30K73 em aplicação no estádio de fenológico $V_{3}$, em função das formulações de nicosulfuron Sanson ${ }^{\circledR}$ e Accent ${ }^{\circledR}$ e diferentes épocas de adubação nitrogenada.

\begin{tabular}{|c|c|c|c|c|c|c|}
\hline \multirow{3}{*}{$\begin{array}{c}\text { Época de } \\
\text { adubação } \\
\text { (DAA) }\end{array}$} & \multicolumn{6}{|c|}{ Altura $(\mathrm{cm})(2 \mathrm{~B}-710)$} \\
\hline & \multicolumn{2}{|c|}{$3 \mathrm{DAA}$} & \multicolumn{2}{|c|}{$7 \mathrm{DAA}$} & \multicolumn{2}{|c|}{$14 \mathrm{DAA}$} \\
\hline & Sanson $^{(}$ & Accent $^{\circledR}$ & Sanson $^{\circledR}$ & Accent $^{\Theta}$ & Sanson $^{\circledR}$ & Accent $^{\circledR}$ \\
\hline 0 & $\left({ }^{\mathrm{ns}}\right) 19,8 \mathrm{aA}$ & $\left({ }^{\mathrm{ns}}\right) 16,4 \mathrm{bA}$ & $\left({ }^{\mathrm{ns}}\right) 29,8 \mathrm{bA}$ & (*) $27,4 \mathrm{aA}$ & (*) $36,0 \mathrm{bA}$ & $(*) 34,0 \mathrm{bA}$ \\
\hline 3 & $\left({ }^{\mathrm{ns}}\right) 18,8 \mathrm{aA}$ & $\left({ }^{\mathrm{ns}}\right) 14,6 \mathrm{aA}$ & $\left({ }^{\mathrm{ns}}\right) 41,2 \mathrm{aA}$ & $\left({ }^{n s}\right) 33,2 \mathrm{aB}$ & $\left({ }^{\mathrm{ns}}\right) 44,4 \mathrm{abA}$ & $\left({ }^{\mathrm{ns}}\right) 51,6 \mathrm{aA}$ \\
\hline 6 & $\left({ }^{\mathrm{ns}}\right) 18,6 \mathrm{aA}$ & $\left({ }^{\mathrm{ns}}\right) 15,4 \mathrm{aA}$ & $\left({ }^{\mathrm{ns}}\right) 42,0 \mathrm{aA}$ & $\left({ }^{\mathrm{ns}}\right) 33,4 \mathrm{aA}$ & $\left({ }^{\mathrm{ns}}\right) 51,8$ a A & $\left({ }^{\mathrm{ns}}\right) 53,4 \mathrm{aA}$ \\
\hline 9 & $\left(\mathrm{~ns}^{\mathrm{n}}\right) 16,4 \mathrm{aA}$ & $\left({ }^{\mathrm{ns}}\right) 16,2 \mathrm{aA}$ & $\left({ }^{\mathrm{ns}}\right) 38,4 \mathrm{aA}$ & $\left({ }^{n s}\right) 34,6 \mathrm{aA}$ & $\left({ }^{n s}\right) 51,0 \mathrm{a} \mathrm{A}$ & $\left({ }^{\mathrm{ns}}\right) 49,0 \mathrm{aA}$ \\
\hline Testemunha & \multicolumn{2}{|c|}{18,2} & \multicolumn{2}{|c|}{36,8} & \multicolumn{2}{|c|}{50,4} \\
\hline $\mathrm{CV}(\%)$ & \multicolumn{2}{|c|}{20,59} & \multicolumn{2}{|c|}{12,05} & \multicolumn{2}{|c|}{16,56} \\
\hline DMS $_{\text {herbicida }}$ & \multicolumn{2}{|c|}{4,53} & \multicolumn{2}{|c|}{5,44} & \multicolumn{2}{|c|}{9,96} \\
\hline \multirow[t]{2}{*}{ DMS $_{\text {adubação }}$} & \multicolumn{2}{|c|}{6,01} & \multicolumn{2}{|c|}{7,22} & \multicolumn{2}{|c|}{13,21} \\
\hline & \multicolumn{6}{|c|}{ Altura $(\mathrm{cm})(30 \mathrm{~K} 73)$} \\
\hline 0 & $\left({ }^{\mathrm{ns}}\right) 18,8 \mathrm{aA}$ & $\left({ }^{\mathrm{ns}}\right) 17,2 \mathrm{aA}$ & $\left({ }^{\mathrm{ns}}\right) 41,1 \mathrm{aA}$ & $\left({ }^{\mathrm{ns}}\right) 35,6 \mathrm{aA}$ & $\left({ }^{\mathrm{ns}}\right) 43,4 \mathrm{aA}$ & $\left({ }^{\mathrm{ns}}\right) 45,4 \mathrm{aA}$ \\
\hline 3 & $\left.{ }^{\mathrm{ns}}\right) 18,6 \mathrm{aA}$ & $\left({ }^{\mathrm{ns}}\right) 17,0 \mathrm{aA}$ & $\left({ }^{\mathrm{ns}}\right) 40,2 \mathrm{aA}$ & $\left({ }^{\mathrm{ns}}\right) 38,6 \mathrm{aA}$ & $\left({ }^{\mathrm{ns}}\right) 45,6 \mathrm{aA}$ & $\left({ }^{\mathrm{ns}}\right) 48,8 \mathrm{aA}$ \\
\hline 6 & $\left.{ }^{\mathrm{ns}}\right) 20,4 \mathrm{aA}$ & $\left({ }^{\mathrm{ns}}\right) 18,0 \mathrm{aA}$ & $\left({ }^{\mathrm{ns}}\right) 42,2 \mathrm{aA}$ & $\left({ }^{\mathrm{ns}}\right) 38,6 \mathrm{aA}$ & $\left({ }^{\mathrm{ns}}\right) 45,2 \mathrm{aA}$ & $\left({ }^{\mathrm{ns}}\right) 45,0 \mathrm{aA}$ \\
\hline 9 & $\left(\mathrm{~ns}^{\mathrm{n}}\right) 18,2 \mathrm{aA}$ & $\left({ }^{\mathrm{ns}}\right) 16,0 \mathrm{aA}$ & $\left({ }^{\mathrm{ns}}\right) 39,0 \mathrm{aA}$ & $\left({ }^{\mathrm{ns}}\right) 38,6 \mathrm{aA}$ & $\left(\mathrm{ns}^{\mathrm{n}}\right) 43,2 \mathrm{aA}$ & $\left({ }^{\mathrm{ns}}\right) 44,4 \mathrm{aA}$ \\
\hline Testemunha & \multicolumn{2}{|c|}{16,8} & \multicolumn{2}{|c|}{39,4} & \multicolumn{2}{|c|}{49,4} \\
\hline $\mathrm{CV}(\%)$ & \multicolumn{2}{|c|}{17,08} & \multicolumn{2}{|c|}{10,14} & \multicolumn{2}{|c|}{8,81} \\
\hline $\mathrm{DMS}_{\text {herbicida }}$ & \multicolumn{2}{|c|}{3,75} & \multicolumn{2}{|c|}{4,95} & \multicolumn{2}{|c|}{5,11} \\
\hline DMS $_{\text {adubação }}$ & \multicolumn{2}{|c|}{4,98} & \multicolumn{2}{|c|}{6,56} & \multicolumn{2}{|c|}{6,78} \\
\hline
\end{tabular}

Médias seguidas da mesma letra minúscula na coluna e maiúscula na linha não diferem entre si pelo teste de Tukey a 5\% de probabilidade. Médias representadas por "*” ou "ns" diferem ou não diferem, respectivamente, da testemunha sem herbicida, segundo o Teste de Dunnett a 5\% de probabilidade.

Para massa seca das raízes do híbrido 2B-710 (Tabela 4), não houve diferença quanto às épocas de adubação nitrogenada. Com relação às formulações de nicosulfuron, somente aos 0 DAAH o herbicida Accent ${ }^{\circledR}$ apresentou menor acúmulo de massa seca de raízes em relação ao Sanson ${ }^{\circledR}$. Todas as combinações entre as épocas de adubação e formulações de nicosulfuron apresentaram interações significativas com a testemunha sem herbicida, sendo caracterizado acúmulo inferior da massa seca da parte aérea e de raízes do híbrido 2B-710. Para o híbrido $30 \mathrm{~K} 73$ não foram constatadas diferenças para massa seca da parte aérea e raízes em nenhuma das combinações entre herbicidas e época de aplicação do adubo nitrogenado em cobertura (Tabela 4). 
Guerra et al.

Tabela 4. Peso médio da massa seca da parte aérea e das raízes dos híbridos de milho 2B-710 e $30 \mathrm{~K} 73$ em aplicação no estádio de fenológico $V_{3}$, em função das formulações de nicosulfuron Sanson ${ }^{\circledR}$ e Accent ${ }^{\circledR}$ e diferentes épocas de adubação nitrogenada.

\begin{tabular}{|c|c|c|c|c|}
\hline \multirow{3}{*}{$\begin{array}{c}\text { Época de } \\
\text { adubação } \\
\text { (DAA) }\end{array}$} & \multicolumn{4}{|c|}{ Massa Seca (g) - híbrido 2B-710 } \\
\hline & \multicolumn{2}{|c|}{ Parte aérea } & \multicolumn{2}{|c|}{ Raízes } \\
\hline & Sanson $^{\circledR}$ & Accent $^{\circledR}$ & Sanson ${ }^{\circledR}$ & Accent $^{\circledR}$ \\
\hline 0 & (*) $0,73 \mathrm{bB}$ & (*) $2,31 \mathrm{bA}$ & $(*) 1,58 \mathrm{aA}$ & $(*) 0,93 \mathrm{aB}$ \\
\hline 3 & (*) $3,00 \mathrm{aA}$ & (*) $3,15 \mathrm{aA}$ & (*) $1,21 \mathrm{aA}$ & (*) $1,22 \mathrm{aA}$ \\
\hline 6 & (*) $2,32 \mathrm{aB}$ & (*) $3,21 \mathrm{aA}$ & (*) $1,00 \mathrm{aA}$ & (*) $1,04 \mathrm{aA}$ \\
\hline 9 & (*) $2,42 \mathrm{aB}$ & (*) $3,27 \mathrm{aA}$ & (*) $1,54 \mathrm{aA}$ & (*) $1,39 \mathrm{aA}$ \\
\hline Testemunha & \multicolumn{2}{|c|}{4,52} & \multicolumn{2}{|c|}{1,76} \\
\hline $\mathrm{CV}(\%)$ & \multicolumn{2}{|c|}{17,42} & \multicolumn{2}{|c|}{14,13} \\
\hline DMS $_{\text {herbicida }}$ & \multicolumn{2}{|c|}{0,82} & \multicolumn{2}{|c|}{0,23} \\
\hline DMS $S_{\text {adubação }}$ & \multicolumn{2}{|c|}{0,62} & \multicolumn{2}{|c|}{0,30} \\
\hline & \multicolumn{4}{|c|}{ Massa Seca (g) - híbrido 30K73 } \\
\hline 0 & $\left({ }^{\mathrm{ns}}\right) 3,18 \mathrm{aA}$ & $\left({ }^{\mathrm{ns}}\right) 2,31 \mathrm{aA}$ & $\left({ }^{\mathrm{ns}}\right) 1,10 \mathrm{aA}$ & $\left({ }^{\mathrm{ns}}\right) 1,35 \mathrm{aA}$ \\
\hline 3 & $\left(^{\mathrm{ns}}\right) 2,72 \mathrm{aA}$ & $\left(^{\mathrm{ns}}\right) 3,35 \mathrm{aA}$ & $\left({ }^{\mathrm{ns}}\right) 1,29 \mathrm{aA}$ & $\left({ }^{\mathrm{ns}}\right) 1,16 \mathrm{aA}$ \\
\hline 6 & $\left({ }^{n s}\right) 3,17 \mathrm{aA}$ & (ns) $3,23 \mathrm{aA}$ & $\left({ }^{\mathrm{ns}}\right) 1,20 \mathrm{aA}$ & $\left({ }^{\mathrm{ns}}\right) 1,34 \mathrm{aA}$ \\
\hline 9 & $\left({ }^{n s}\right) 2,64 \mathrm{aA}$ & $\left({ }^{\mathrm{ns}}\right) 3,09 \mathrm{aA}$ & $\left({ }^{\mathrm{ns}}\right) 1,06 \mathrm{aA}$ & $\left({ }^{\mathrm{ns}}\right) 1,00 \mathrm{aA}$ \\
\hline Testemunha & \multicolumn{2}{|c|}{2,98} & \multicolumn{2}{|c|}{$1,11^{-}$} \\
\hline $\mathrm{CV}(\%)$ & \multicolumn{2}{|c|}{31,80} & \multicolumn{2}{|c|}{35,59} \\
\hline DMS $_{\text {herbicida }}$ & \multicolumn{2}{|c|}{1,26} & \multicolumn{2}{|c|}{0,58} \\
\hline $\mathrm{DMS}_{\text {adubacão }}$ & \multicolumn{2}{|c|}{1,67} & \multicolumn{2}{|c|}{0,77} \\
\hline
\end{tabular}

Médias seguidas da mesma letra minúscula na coluna e maiúscula na linha não diferem entre si pelo teste de Tukey a $5 \%$ de probabilidade. Médias representadas por “*” ou "ns" diferem ou não diferem, respectivamente, da testemunha sem herbicida, segundo o Teste de Dunnett a 5\% de probabilidade.

\section{Híbridos em Estádio $V_{6}$}

No estádio de $\mathrm{V}_{6}$, os híbridos 2B-710 e 30K73 apresentaram maiores sintomas de fitointoxicação quando a adubação nitrogenada foi realizada aos 3 DAAH (Tabela 5). Para 2B710, as formulações Sanson $^{\circledR}$ e Accent $^{\circledR}$ proporcionaram sintomas de injúrias semelhantes na parte aérea das plantas, caracterizados por leve clorose e encarquilhamento das bordas da lâmina foliar, não ultrapassando $12,4 \%$ de danos (7 DAA).

Para teor de clorofila nas folhas do híbrido 2B-710 em $\mathrm{V}_{6}$ na avaliação realizada aos 3 DAA, foi constatado redução inicial desta variável quando se empregou a formulação Sanson ${ }^{\circledR}$ em relação ao Accent ${ }^{\circledR}$ para adubação nitrogenada efetuada aos 0 DAAH. Em relação a testemunha, a formulação Accent ${ }^{\circledR}$ também foi inferior para adubação aos 3 DAAH (Tabela 6). O híbrido 30K73 não apresentou diferenças entre formulações, épocas de adubação nitrogenada em cobertura.

Os híbridos 2B-710 e 30K73 não apresentaram diferenças para altura das plantas quando submetidos à aplicação de formulações de nicosulfuron em estádio $V_{6}$ e realizado adubação nitrogenada em diferentes períodos (Tabela 7). Apenas foi observado para o híbrido 2B-710 crescimento superior do Sanson ${ }^{\circledR}$ em relação ao Accent ${ }^{\circledR}$ para adubação nitrogenada efetuada aos 3 DAAH, na avaliação realizada aos 7 DAA 
Guerra et al.

Tabela 5. Fitointoxicação visual (\%) dos híbridos de milho 2B-710 e 30K73 em aplicação no estádio de fenológico $\mathrm{V}_{6}$, em função das formulações de nicosulfuron Sanson ${ }^{\circledR}$ e Accent ${ }^{\circledR}$ e diferentes épocas de adubação nitrogenada.

\begin{tabular}{|c|c|c|c|c|c|c|}
\hline \multirow{4}{*}{$\begin{array}{c}\text { Época de } \\
\text { adubação } \\
\text { (DAA) }\end{array}$} & \multicolumn{6}{|c|}{ Fitointoxicação (\%) (2B-710) } \\
\hline & \multicolumn{2}{|c|}{3 DAA } & \multicolumn{2}{|c|}{7 DAA } & \multicolumn{2}{|c|}{$14 \mathrm{DAA}$} \\
\hline & \multicolumn{2}{|c|}{ Formulações } & \multicolumn{2}{|c|}{ Formulações } & \multicolumn{2}{|c|}{ Formulações } \\
\hline & Sanson $^{\circledR}$ & Accent $^{\circledR}$ & Sanson $^{\circledR}$ & Accent $^{\circ}$ & Sanson $\left.^{(}\right)$ & Accent $^{B}$ \\
\hline 0 & 0,0 & 3,2 & 0,0 & 0,0 & 0,0 & 0,0 \\
\hline 3 & 6,4 & 5,8 & 10,4 & 12,4 & 10,8 & 7,6 \\
\hline 6 & 4,6 & 6,6 & 0,0 & 0,0 & 0,0 & 0,0 \\
\hline 9 & 4,6 & 5,4 & 0,0 & 0,0 & 0,0 & 0,0 \\
\hline \multirow[t]{2}{*}{ Testemunha } & \multicolumn{2}{|c|}{0,0} & \multicolumn{2}{|c|}{0,0} & \multicolumn{2}{|c|}{0,0} \\
\hline & \multicolumn{6}{|c|}{ Fitointoxicação (\%) (30K73) } \\
\hline 0 & 0,0 & 0,0 & 0,0 & 0,0 & 0,0 & 0,0 \\
\hline 3 & 2,4 & 14,2 & 18,4 & 30,6 & 18,6 & 32,0 \\
\hline 6 & 0,0 & 0,0 & 0,0 & 0,0 & 0,0 & 0,0 \\
\hline 9 & 0,0 & 0,0 & 0,0 & 0,0 & 0,0 & 0,0 \\
\hline Testemunha & \multicolumn{2}{|c|}{0,0} & \multicolumn{2}{|c|}{0,0} & \multicolumn{2}{|c|}{0,0} \\
\hline
\end{tabular}

Tabela 6. Teor de clorofila nas folhas (SPAD) dos híbridos de milho 2B-710 e 30K73 em aplicação no estádio de fenológico $\mathrm{V}_{6}$, em função das formulações de nicosulfuron Sanson ${ }^{\circledR} \mathrm{e}$ Accent ${ }^{\circledR}$ e diferentes épocas de adubação nitrogenada.

\begin{tabular}{|c|c|c|c|c|c|c|}
\hline \multirow{3}{*}{$\begin{array}{c}\text { Época de } \\
\text { adubação } \\
\text { (DAA) }\end{array}$} & \multicolumn{6}{|c|}{ Teor de clorofila (SPAD) - híbrido 2B-710 } \\
\hline & \multicolumn{2}{|c|}{3 DAA } & \multicolumn{2}{|c|}{7 DAA } & \multicolumn{2}{|c|}{14 DAA } \\
\hline & Sanson $^{\circledR}$ & Accent $^{\circledR}$ & Sanson $^{\circledR}$ & Accent $^{\circledR}$ & Sanson ${ }^{\circledR}$ & Accent $^{\circledR}$ \\
\hline 0 & (*) $31,7 \mathrm{aB}$ & $\left({ }^{\mathrm{ns}}\right) 35,4 \mathrm{aA}$ & (ns) $31,7 \mathrm{aA}$ & $\left({ }^{\mathrm{ns}}\right) 35,8 \mathrm{aA}$ & $\left({ }^{n s}\right) 31,1 \mathrm{aA}$ & $\left({ }^{\mathrm{ns}}\right) 30,9 \mathrm{aA}$ \\
\hline 3 & $\left({ }^{\mathrm{ns}}\right) 33,8 \mathrm{aA}$ & (*) $31,8 \mathrm{aA}$ & $\left(^{\mathrm{ns}}\right) 37,8 \mathrm{aA}$ & $\left({ }^{\mathrm{ns}}\right) 36,4 \mathrm{aA}$ & $\left.{ }^{\mathrm{ns}}\right) 31,6 \mathrm{aA}$ & $\left({ }^{\mathrm{ns}}\right) 39,0 \mathrm{aA}$ \\
\hline 6 & $\left({ }^{\mathrm{ns}}\right) 33,7 \mathrm{aA}$ & $\left({ }^{\mathrm{ns}}\right) 33,5 \mathrm{aA}$ & $\left({ }^{\mathrm{ns}}\right) 38,8 \mathrm{aA}$ & $\left({ }^{\mathrm{ns}}\right) 37,4 \mathrm{aA}$ & $\left({ }^{\mathrm{ns}}\right) 39,3 \mathrm{aA}$ & $\left({ }^{\mathrm{ns}}\right) 38,9 \mathrm{aA}$ \\
\hline 9 & $\left({ }^{\mathrm{ns}}\right) 33,2 \mathrm{aA}$ & $\left({ }^{\mathrm{ns}}\right) 33,4 \mathrm{aA}$ & $\left({ }^{\mathrm{ns}}\right) 37,5 \mathrm{aA}$ & $\left({ }^{\mathrm{ns}}\right) 35,5 \mathrm{aA}$ & $\left({ }^{\mathrm{ns}}\right) 27,4 \mathrm{aA}$ & $\left({ }^{\mathrm{ns}}\right) 33,2 \mathrm{aA}$ \\
\hline Testemunha & \multicolumn{2}{|c|}{35,99} & \multicolumn{2}{|c|}{40,05} & \multicolumn{2}{|c|}{39,02} \\
\hline $\mathrm{CV}(\%)$ & \multicolumn{2}{|c|}{6,88} & \multicolumn{2}{|c|}{17,73} & \multicolumn{2}{|c|}{30,16} \\
\hline DMS $S_{\text {herbicida }}$ & \multicolumn{2}{|c|}{2,97} & \multicolumn{2}{|c|}{8,73} & \multicolumn{2}{|c|}{13,37} \\
\hline $\mathrm{DMS}_{\text {adubação }}$ & \multicolumn{2}{|c|}{3,93} & \multicolumn{2}{|c|}{11,10} & \multicolumn{2}{|c|}{17,74} \\
\hline & \multicolumn{6}{|c|}{ Teor de clorofila (SPAD) - híbrido 30K73 } \\
\hline 0 & $\left({ }^{\mathrm{ns}}\right) 39,2 \mathrm{aA}$ & $\left({ }^{\mathrm{ns}}\right) 38,4 \mathrm{aA}$ & $\left({ }^{\mathrm{ns}}\right) 39,1 \mathrm{aA}$ & $\left({ }^{\mathrm{ns}}\right) 39,9 \mathrm{aA}$ & $\left({ }^{\mathrm{ns}}\right) 41,3 \mathrm{aA}$ & $\left({ }^{\mathrm{ns}}\right) 40,8 \mathrm{aA}$ \\
\hline 3 & $\left({ }^{\mathrm{ns}}\right) 38,7 \mathrm{aA}$ & $\left({ }^{\mathrm{ns}}\right) 37,8 \mathrm{aA}$ & $\left({ }^{\mathrm{ns}}\right) 40,6 \mathrm{aA}$ & $\left({ }^{\mathrm{ns}}\right) 41,8 \mathrm{aA}$ & $\left({ }^{\mathrm{ns}}\right) 41,0 \mathrm{aA}$ & $\left({ }^{\mathrm{ns}}\right) 39,1 \mathrm{aA}$ \\
\hline 6 & $\left({ }^{\mathrm{ns}}\right) 39,1 \mathrm{aA}$ & $\left({ }^{\mathrm{ns}}\right) 38,5 \mathrm{aA}$ & $\left({ }^{\mathrm{ns}}\right) 40,9 \mathrm{aA}$ & $\left({ }^{\mathrm{ns}}\right) 42,1 \mathrm{aA}$ & $\left({ }^{\mathrm{ns}}\right) 41,4 \mathrm{aA}$ & $\left({ }^{\mathrm{ns}}\right) 41,4 \mathrm{aA}$ \\
\hline 9 & $\left({ }^{n s}\right) 36,8 \mathrm{aA}$ & $\left({ }^{\mathrm{ns}}\right) 38,9 \mathrm{aA}$ & $\left({ }^{\mathrm{ns}}\right) 40,5 \mathrm{aA}$ & $\left({ }^{n s}\right) 41,5 \mathrm{aA}$ & $\left({ }^{\mathrm{ns}}\right) 40,8 \mathrm{aA}$ & $\left({ }^{\mathrm{ns}}\right) 39,1 \mathrm{aA}$ \\
\hline Testemunha & \multicolumn{2}{|c|}{40,22} & \multicolumn{2}{|c|}{40,88} & \multicolumn{2}{|c|}{41,47} \\
\hline $\mathrm{CV}(\%)$ & \multicolumn{2}{|c|}{5,05} & \multicolumn{2}{|c|}{$\overline{5,00}$} & \multicolumn{2}{|c|}{5,05} \\
\hline DMS ${ }_{\text {herbicida }}$ & \multicolumn{2}{|c|}{2,50} & \multicolumn{2}{|c|}{2,62} & \multicolumn{2}{|c|}{2,64} \\
\hline $\mathrm{DMS}_{\text {adubação }}$ & \multicolumn{2}{|c|}{3,32} & \multicolumn{2}{|c|}{3,47} & \multicolumn{2}{|c|}{3,50} \\
\hline
\end{tabular}


Guerra et al.

Diferentemente do estágio $\mathrm{V}_{3}$, o híbrido 2B-710 em estádio $\mathrm{V}_{6}$ apresentou maior seletividade às formulações Sanson ${ }^{\circledR}$ e Accent $^{\circledR}$ quando associado à adubação realizada aos 0 DAAH. Entretanto, no estádio $\mathrm{V}_{6}$ o híbrido 30K73 com adubação nitrogenada aos 3 DAAH, apresentou maior sensibilidade para formulação Accent $^{\circledR}$, causando níveis médios de fitointoxicação de $31,3 \%$ aos 7 e 14 DAA.

Com exceção da época de adubação nitrogenada aos 3 DAAH, não foram constatadas diferenças significativas da massa seca da parte aérea e raízes dos híbridos 2B710 e $30 \mathrm{~K} 73$ em estádio $V_{6}$, para formulações e épocas de adubação nitrogenada, assim como em relação à testemunha sem herbicida (Tabela 8). Esses resultados corroboram com as observações de fitointoxicação caracterizadas para adubação nitrogenada aos 3 DAAH, e indicam que o híbrido $2 \mathrm{~B}-710$ em estádio $\mathrm{V}_{6}$ apresentou maior tolerância aos efeitos da combinações entre as épocas de adubação e formulações de nicosulfuron em relação ao estádio $\mathrm{V}_{3}$.

Tabela 7. Altura média $(\mathrm{cm})$ dos híbridos de milho 2B-710 e 30K73 em aplicação no estádio de fenológico $V_{6}$, em função das formulações de nicosulfuron Sanson ${ }^{\circledR}$ e Accent $^{\circledR}$ e diferentes épocas de adubação nitrogenada.

\begin{tabular}{|c|c|c|c|c|c|c|}
\hline \multirow{3}{*}{$\begin{array}{c}\text { Época de } \\
\text { adubação } \\
\text { (DAA) }\end{array}$} & \multicolumn{6}{|c|}{ Altura $(\mathrm{cm})(2 \mathrm{~B}-710)$} \\
\hline & \multicolumn{2}{|c|}{3 DAA } & \multicolumn{2}{|c|}{7 DAA } & \multicolumn{2}{|c|}{14 DAA } \\
\hline & Sanson $^{\circledR}$ & Accent $\left.^{(}\right)$ & Sanson $^{\circledR}$ & Accent $^{\circledR}$ & Sanson $\left.^{(}\right)$ & Accent $^{\circledR}$ \\
\hline 0 & $\left({ }^{\mathrm{ns}}\right) 29,8 \mathrm{aA}$ & $\left({ }^{\mathrm{ns}}\right) 25,0 \mathrm{aA}$ & $\left({ }^{\mathrm{ns}}\right) 50,6 \mathrm{aA}$ & $\left({ }^{\mathrm{ns}}\right) 48,2 \mathrm{aA}$ & $\left({ }^{\mathrm{ns}}\right) 66,9 \mathrm{aA}$ & $\left({ }^{\mathrm{ns}}\right) 66,6 \mathrm{aA}$ \\
\hline 3 & $\left({ }^{\mathrm{ns}}\right) 26,8 \mathrm{aA}$ & $\left({ }^{\mathrm{ns}}\right) 24,2 \mathrm{aA}$ & $\left({ }^{\mathrm{ns}}\right) 51,8 \mathrm{aA}$ & $\left({ }^{\mathrm{ns}}\right) 46,2 \mathrm{aB}$ & $\left({ }^{\mathrm{ns}}\right) 66,4 \mathrm{aA}$ & $\left({ }^{\mathrm{ns}}\right) 66,4 \mathrm{aA}$ \\
\hline 6 & $\left({ }^{n s}\right) 29,2 \mathrm{aA}$ & $\left({ }^{\mathrm{ns}}\right) 25,8 \mathrm{aA}$ & $\left({ }^{\mathrm{ns}}\right) 54,0 \mathrm{aA}$ & $\left({ }^{\mathrm{ns}}\right) 47,2 \mathrm{aB}$ & $\left({ }^{\mathrm{ns}}\right) 69,4 \mathrm{aA}$ & $\left({ }^{\mathrm{ns}}\right) 67,9 \mathrm{aA}$ \\
\hline 9 & $\left({ }^{\mathrm{ns}}\right) 26,2 \mathrm{aA}$ & $\left({ }^{\mathrm{ns}}\right) 25,0 \mathrm{aA}$ & $\left({ }^{\mathrm{ns}}\right) 54,0 \mathrm{aA}$ & $\left({ }^{\mathrm{ns}}\right) 47,6 \mathrm{aB}$ & $\left({ }^{\mathrm{ns}}\right) 69,4 \mathrm{aA}$ & $\left({ }^{\mathrm{ns}}\right) 68,6 \mathrm{aA}$ \\
\hline Testemunha & \multicolumn{2}{|c|}{29,2} & \multicolumn{2}{|c|}{49,6} & \multicolumn{2}{|c|}{69,4} \\
\hline $\mathrm{CV}(\%)$ & \multicolumn{2}{|c|}{14,50} & \multicolumn{2}{|c|}{6,29} & \multicolumn{2}{|c|}{4,66} \\
\hline DMS $_{\text {herbicida }}$ & \multicolumn{2}{|c|}{4,99} & \multicolumn{2}{|c|}{4,03} & \multicolumn{2}{|c|}{3,91} \\
\hline DMS $_{\text {adubação }}$ & \multicolumn{2}{|c|}{6,61} & \multicolumn{2}{|c|}{5,34} & \multicolumn{2}{|c|}{5,18} \\
\hline & \multicolumn{6}{|c|}{ Altura $(\mathrm{cm})(30 \mathrm{~K} 73)$} \\
\hline$\overline{0}$ & $\left({ }^{\mathrm{ns}}\right) 22,5 \mathrm{aA}$ & $\left({ }^{\mathrm{ns}}\right) 30,4 \mathrm{aA}$ & $\left({ }^{\mathrm{ns}}\right) 50,8 \mathrm{aA}$ & $\left({ }^{\mathrm{ns}}\right) 48,8 \mathrm{aA}$ & $\left({ }^{\mathrm{ns}}\right) 60,2 \mathrm{aA}$ & $\left({ }^{\mathrm{ns}}\right) 62,8 \mathrm{aA}$ \\
\hline 3 & $\left({ }^{\mathrm{ns}}\right) 29,0 \mathrm{aA}$ & $\left({ }^{\mathrm{ns}}\right) 28,4 \mathrm{aA}$ & $\left({ }^{\mathrm{ns}}\right) 50,8 \mathrm{aA}$ & $\left({ }^{\mathrm{ns}}\right) 53,8 \mathrm{aA}$ & $\left({ }^{\mathrm{ns}}\right) 60,0 \mathrm{aA}$ & $\left({ }^{\mathrm{ns}}\right) 63,2 \mathrm{aA}$ \\
\hline 6 & $\left({ }^{\mathrm{ns}}\right) 28,2 \mathrm{aA}$ & $\left({ }^{\mathrm{ns}}\right) 28,8 \mathrm{aA}$ & $\left(^{\mathrm{ns}}\right) 54,8 \mathrm{aA}$ & $\left({ }^{\mathrm{ns}}\right) 52,2 \mathrm{aA}$ & $\left({ }^{\mathrm{ns}}\right) 59,6 \mathrm{aA}$ & $\left({ }^{\mathrm{ns}}\right) 63,4 \mathrm{aA}$ \\
\hline 9 & $\left({ }^{\mathrm{ns}}\right) 28,2 \mathrm{aA}$ & $\left({ }^{\mathrm{ns}}\right) 26,8 \mathrm{aA}$ & $\left({ }^{\mathrm{ns}}\right) 51,0 \mathrm{aA}$ & $\left({ }^{\mathrm{ns}}\right) 52,8 \mathrm{aA}$ & $\left({ }^{n s}\right) 60,4 \mathrm{aA}$ & $\left({ }^{\mathrm{ns}}\right) 62,9 \mathrm{aA}$ \\
\hline Testemunha & \multicolumn{2}{|c|}{25,0} & \multicolumn{2}{|c|}{50,2} & \multicolumn{2}{|c|}{67,4} \\
\hline $\mathrm{CV}(\%)$ & \multicolumn{2}{|c|}{15,29} & \multicolumn{2}{|c|}{7,26} & \multicolumn{2}{|c|}{4,12} \\
\hline DMS $_{\text {herbicida }}$ & \multicolumn{2}{|c|}{5,39} & \multicolumn{2}{|c|}{4,77} & \multicolumn{2}{|c|}{3,20} \\
\hline $\mathrm{DMS}_{\text {adubação }}$ & \multicolumn{2}{|c|}{7,15} & \multicolumn{2}{|c|}{6,32} & \multicolumn{2}{|c|}{4,25} \\
\hline
\end{tabular}

Médias seguidas da mesma letra minúscula na coluna e maiúscula na linha não diferem entre si pelo teste de Tukey a 5\% de probabilidade. Médias representadas por "*”" ou "ns" diferem ou não diferem, respectivamente, da testemunha sem herbicida, segundo o Teste de Dunnett a 5\% de probabilidade.

De forma geral, os resultados obtidos indicam que os híbridos 2B-710 e 30K73 apresentaram comportamentos distintos quanto à seletividade de formulações do herbicida nicosulfuron em interação com adubação nitrogenada. Segundo Morton \& Harvey
(1992), a tolerância dos híbridos de milho à herbicidas sulfoniluréias é bastante variável, podendo ser elevada para alguns e reduzida para outros. Híbridos considerados tolerantes a estes herbicidas podem apresentar sensibilidade, dependendo do estádio de 
Guerra et al.

desenvolvimento da planta, do ambiente e da herbicida nicosulfuron não interferiram na dose utilizada. Nesse sentido, Nicolai et al. altura de plantas, massa de 1000 grãos e (2006) verificaram que diferentes épocas de rendimento.

adubação nitrogenada em interação com o

Tabela 8. Peso médio da massa seca da parte aérea e das raízes dos híbridos de milho 2B-710 e $30 \mathrm{~K} 73$ em aplicação no estádio de fenológico $\mathrm{V}_{6}$, em função das formulações de nicosulfuron Sanson ${ }^{\circledR}$ e Accent ${ }^{\circledR}$ e diferentes épocas de adubação nitrogenada.

\begin{tabular}{|c|c|c|c|c|}
\hline \multirow{3}{*}{$\begin{array}{c}\text { Época de } \\
\text { adubação } \\
\text { (DAA) }\end{array}$} & \multicolumn{4}{|c|}{ Massa Seca (g) - híbrido 2B-710 } \\
\hline & \multicolumn{2}{|c|}{ Parte aérea } & \multicolumn{2}{|c|}{ Raízes } \\
\hline & Sanson $^{\circledR}$ & Accent $^{\circledR}$ & Sanson $^{(\otimes)}$ & Accent $^{\circledR}$ \\
\hline 0 & $\left({ }^{\mathrm{ns}}\right) 11,19 \mathrm{aA}$ & ${ }^{\left({ }^{n s}\right)} 11,67 \mathrm{abA}$ & $\left({ }^{\mathrm{ns}}\right) 2,80 \mathrm{aA}$ & $\left({ }^{\mathrm{ns}}\right) 2,88 \mathrm{aA}$ \\
\hline 3 & $\left({ }^{\mathrm{ns}}\right) 14,24 \mathrm{aA}$ & $\left(^{*}\right) 8,48 \mathrm{bA}$ & $\left({ }^{n s}\right) 3,19 \mathrm{aA}$ & $\left({ }^{\mathrm{ns}}\right) 1,50 \mathrm{aB}$ \\
\hline 6 & $\left({ }^{\mathrm{ns}}\right) 14,46 \mathrm{aA}$ & $\left({ }^{\mathrm{ns}}\right) 16,68 \mathrm{aA}$ & $\left({ }^{\mathrm{ns}}\right) 2,82 \mathrm{aA}$ & $\left({ }^{\mathrm{ns}}\right) 2,00 \mathrm{aA}$ \\
\hline 9 & $\left(\mathrm{~ns}^{\mathrm{n}}\right) 17,77 \mathrm{aA}$ & $\left({ }^{\mathrm{ns}}\right) 12,84 \mathrm{abA}$ & $\left({ }^{\mathrm{ns}}\right) 2,72 \mathrm{aA}$ & $\left({ }^{\mathrm{ns}}\right) 2,58 \mathrm{aA}$ \\
\hline Testemunha & \multicolumn{2}{|c|}{16,29} & \multicolumn{2}{|c|}{2,33} \\
\hline $\mathrm{CV}(\%)$ & \multicolumn{2}{|c|}{30,95} & \multicolumn{2}{|c|}{28,59} \\
\hline DMS $_{\text {herbicida }}$ & \multicolumn{2}{|c|}{5,46} & \multicolumn{2}{|c|}{0,97} \\
\hline DMS $_{\text {adubação }}$ & \multicolumn{2}{|c|}{7,24} & \multicolumn{2}{|c|}{1,29} \\
\hline & \multicolumn{4}{|c|}{ Massa Seca (g) - híbrido 30K73 } \\
\hline 0 & $\left({ }^{\mathrm{ns}}\right) 14,54 \mathrm{aA}$ & $\left({ }^{\mathrm{ns}}\right) 12,81 \mathrm{aA}$ & $\left({ }^{\mathrm{ns}}\right) 2,61 \mathrm{aA}$ & $\left({ }^{\mathrm{ns}}\right) 3,02 \mathrm{aA}$ \\
\hline 3 & $\left.{ }^{(\mathrm{ns}}\right) 13,44 \mathrm{aA}$ & $\left({ }^{\mathrm{ns}}\right) \quad 9,84 \mathrm{aA}$ & $\left({ }^{n s}\right) 3,06$ aA & $\left({ }^{\mathrm{ns}}\right) 2,10 \mathrm{aB}$ \\
\hline 6 & $\left.\left.{ }^{(\mathrm{n}}\right)^{2}\right) 15,11 \mathrm{aA}$ & $\left({ }^{\mathrm{ns}}\right) 13,04 \mathrm{aA}$ & $\left({ }^{\mathrm{ns}}\right) 3,47 \mathrm{aA}$ & $\left({ }^{\mathrm{ns}}\right) 3,00 \mathrm{aA}$ \\
\hline 9 & $\left({ }^{\mathrm{ns}}\right) 12,83 \mathrm{aA}$ & $\left({ }^{\mathrm{ns}}\right) 12,72 \mathrm{aA}$ & $\left({ }^{n s}\right) 2,58 \mathrm{aA}$ & $\left({ }^{\mathrm{ns}}\right) 2,15 \mathrm{aA}$ \\
\hline Testemunha & \multicolumn{2}{|c|}{15,20} & \multicolumn{2}{|c|}{3,34} \\
\hline $\mathrm{CV}(\%)$ & \multicolumn{2}{|c|}{25,45} & \multicolumn{2}{|c|}{25,75} \\
\hline DMS $_{\text {herbicida }}$ & \multicolumn{2}{|c|}{4,33} & \multirow{2}{*}{\multicolumn{2}{|c|}{$\begin{array}{l}0,93 \\
1,23\end{array}$}} \\
\hline DMS $_{\text {adubacão }}$ & \multicolumn{2}{|c|}{5,75} & & \\
\hline
\end{tabular}

Médias seguidas da mesma letra minúscula na coluna e maiúscula na linha não diferem entre si pelo teste de Tukey a 5\% de probabilidade. Médias representadas por "*” ou "ns" diferem ou não diferem, respectivamente, da testemunha sem herbicida, segundo o Teste de Dunnett a 5\% de probabilidade.

\section{Conclusões}

Com base no que foi exposto, concluise que os híbridos de milho 2B-710 e $30 \mathrm{~K} 73$ apresentaram seletividade variável quanto à aplicação de nicosulfuron, sendo que os maiores efeitos e sintomas da fitointoxicação ocorreram quando a adubação nitrogenada foi realizada em cobertura no mesmo dia da aplicação da formulação Sanson ${ }^{\circledR}$ para o estádio $V_{3}$ e aos três dias da aplicação de Accent ${ }^{\circledR}$ para o estádio $\mathrm{V}_{6}$.

\section{Referências}

CANTARELLA, H.; DUARTE, A. P.; ANDRADE, C. A. Manejo de nitrogênio e de matéria orgânica em milho no sistema plantio direto. In: FANCELLI, A. L.; DOURADO NETO, D. (Eds.). Milho: tecnologia \& produção. Piracicaba: ESALQ, 2005. p.59-82.

CHRISTOFFOLETI, P.J.; MENDONÇA, C.G. Controle de plantas daninhas na cultura de milho: enfoque atual. In: FANCELLI, A.L.; DOURADO-NETO, D. (Coords.). Milho: tecnologia e produtividade. Piracicaba: ESALQ, 2001. p.60-95.

COSTA, F.M.P. et al. Nitrogênio e produtividade de grãos de milho. In: FANCELLI, A. L.; DOURADO NETO, D. 
Guerra et al.

(Eds.). Milho: tecnologia \& produção. PEIXOTO, C.M.; RAMOS, A.A. Milho: Piracicaba: ESALQ., 2005. p. 118-128.

DEVINE, M.; DUKE, S.O.; FEDTKE, C. Physiology of herbicide action. Englewood Cliffs: PTR Prentice Hall, 1993. 441 p.

FANCELLI, A.L.; DOURADO-NETO, D. Produção de milho. Guaíba: Agropecuária, 2000. 360 p.

LÓPEZ OVEJERO, R.F. et al. Manejo de plantas daninhas na cultura do milho. In: FANCELLI, A.L.; DOURADO NETO, D. (Eds.). Milho: estratégias de manejo para alta produtividade. Piracicaba: ESALQ, 2003a. p.47-79.

LÓPEZ-OVEJERO, R.F. et al. Seletividade de herbicidas para a cultura do milho (Zea mays) aplicado em diferentes estádios fenológicos da cultura. Planta Daninha. v.21, n.3, p.413-419, 2003b.

MASCARENHAS, M.H.T. Eficácia e seletividade do mesotrione + (atrazine+ óleo vegetal) sobre híbridos de milho associado à época de aplicação do fertilizante nitrogenado. Boletim Informativo Sociedade Brasileira da Ciência das Plantas Daninhas, v. 10, p. 132, 2004. (Suplemento).

MORTON, C.A., HARVEY, R.G. Sweet corn (Zea mays) hybrid tolerance to nicosulfuron. Weed Technology, v.6, n.1, p.91-96, 1992.

NICOLAI, M. et al. Efeitos da adubação nitrogenada em cobertura sobre a seletividade de herbicidas à cultura do milho. Planta Daninha, v.24, n.2, p.279-286, 2006. manejo de herbicidas. Caderno técnico, Cultivar grandes culturas. n. 42, Edição Especial Pioneer, 2002.

RIZZARDI, M.A. et al. Controle de plantas daninhas em milho em função da época de aplicação de nitrogênio. Planta Daninha, v.26, n.1, p.113-121, 2008.

RODRIGUES, B.N.; ALMEIDA, F.S. Guia de herbicidas. $5^{\text {a }}$ edição. Londrina: Editora dos autores. 2005. 592p.

SILVA, A.A. et al. Manejo de plantas daninhas na cultura do milho. In: GALVÃO, J. C. C.; MIRANDA, G. V. Tecnologias de produção do milho. Viçosa: UFV. 2004. p.269 - 310.

SILVA, A.A.; SILVA, J.F. Tópicos em manejo de plantas daninhas. Viçosa, MG: UFV. 2007. 367 p.

SOCIEDADE BRASILEIRA DA CIÊNCIA DAS PLANTAS DANINHAS. Procedimentos para instalação, avaliação e análise de experimentos com herbicidas. Londrina: SBCPD, 1995. 42p.

SPADER, V.; VIDAL, R.A. Seletividade e dose de injúria econômica de nicosulfuron aplicado em diferentes estádios de desenvolvimento da cultura do milho. Ciência Rural, v.31, n.6, p.929-934, 2001.

VICTORIA FILHO, R. Estratégias de manejo de plantas daninhas. In: ZAMBOLIN, L. et al. (ed). O que os engenheiros agrônomos devem saber para orientar o uso de produtos fitossanitários. 3 ed.rev. amp.. Viçosa: UFV, 2008. p.397- 458. 